Tropical Journal of Pharmaceutical Research August 2010; 9 (4): 355-363

(C) Pharmacotherapy Group, Faculty of Pharmacy, University of Benin,

Benin City, 300001 Nigeria.

All rights reserved.

Research Article

Available online at http://www.tjpr.org

\title{
Development and Evaluation of Ibuprofen Transdermal Gel Formulations
}

\author{
Bazigha K Abdul Rasool ${ }^{1 *}$, Eman F Abu-Gharbieh", Sahar A \\ Fahmy ${ }^{2}$, Heyam S Saad ${ }^{1}$ and Saeed A Khan ${ }^{1}$ \\ ${ }^{1}$ Dubai Pharmacy College, Dubai, UAE, ${ }^{2}$ Helwan University, Helwan, Egypt
}

\begin{abstract}
Purpose: To develop an ibuprofen transdermal gel with a capability for both topical and systemic drug delivery.

Methods: Ibuprofen gel formulations, incorporating various permeation enhancers, were prepared using chitosan as a gelling agent. The formulations were examined for their in vitro characteristics including viscosity, $\mathrm{pH}$ and drug release as well as in vivo pharmacological activities. Carrageenan-induced rat paw oedema model was used for the evaluation of their analgesic and anti-inflammatory activities. A commercial ibuprofen gel product (lbutop ${ }^{\circledR}$ ) was used as a reference.

Results: The formulations containing $5 \%$ of either menthol or glycerol as permeation enhancers gave drug release patterns comparable to that of the reference product. Propanol increased the apparent viscosity of the test gels to the same extent as that of the reference. Drug release from the formulations fitted best to the Higuchi model. A significant in vivo analgesic effect was produced by the test formulations containing $5 \%$ menthol and $20 \%$ propylene glycol and the effect was superior to that obtained with the reference product. However, no significant anti-inflammatory activity was exerted by any of the test gel formulations ( $p>0.05)$.

Conclusion: Ibuprofen gel preparations containing $5 \%$ menthol and $20 \%$ propylene glycol, respectively, exhibited pronounced analgesic activity and could be further developed for topical and systemic delivery of ibuprofen.
\end{abstract}

Keywords: Transdermal gel, Chitosan, Ibuprofen, Menthol, Propylene glycol, Penetration enhancer 


\section{INTRODUCTION}

Drug delivery through the skin has been a promising concept for a long time because skin is easy to access, has a large surface area with vast exposure to the circulatory and lymphatic networks and the route is noninvasive. [1]. Transdermal delivery is of great importance for drugs that may cause systemic side effects such as non steroidal anti-inflammatory drugs (NSAIDs)[2].

Ibuprofen is an effective non-steroidal antiinflammatory drug (NSAID) often used in the treatment of acute and chronic arthritic conditions [3]. There is great interest to develop non-oral dosage forms of ibuprofen to minimize its gastric side effects and to provide relatively consistent drug levels at the application site for prolonged periods [2]. Transdermal delivery of ibuprofen using various formulations has been described in the literature [4,5]. However, effective permeation of ibuprofen through skin is difficult to achieve due to its intrinsically poor permeability, though this is relatively good compared to other commonly used NSAIDs [6]. However, skin permeation enhancers could be used to improve drug skin penetration [7].

Chitosan has unique non-toxic and antimicrobial characteristics, along with a solution property and other attributes which make it an attractive biopolymer for many pharmaceutical and biomedical applications via different routes of administration [8]. It has been is used in gel formulations to prolong drug residence time as well as reduce the application frequency and amount of drug administered, factors which might improve patient compliance and acceptance [9].

Carrageenan-induced paw oedema in mice has been widely used for the evaluation of analgesic and anti-inflammatory activities of drugs since the relative potency found for most drugs tend to reflect clinical experience [10].
The aim of this study was to develop suitable transdermal gel formulations of ibuprofen using chitosan polymer as a gelling agent, and various skin permeation enhancers

\section{EXPERIMENTAL}

\section{Materials}

Ibuprofen was kindly provided by Gulf Pharmaceutical Industries, UAE while Ibutop ${ }^{\circledR}$ gel $5 \%$ was obtained from Deutsche Chefaro Pharma $\mathrm{GmbH}$, Germany. Other materials used were chitosan (MW: $2.5 \times 103 \mathrm{kDa}$, Biotech. Co. Ltd Korea), a synthetic hydrophilic membrane (GH Polypro filter, Waters Corporation, Philadelphia, PA) with a pore size of $0.45 \mathrm{~mm}$, menthol (Pacegrone, England), glycerol (E. Merck, Germany), propanol (ADWIC, Egypt), oleic acid (AIMIA, Australia), propylene glycol (Fluka Chemie, Switzerland), polyethylene glycol PEG400 (Sigma, Germany), potassium dihydrogen phosphate (E. Merck, Germany), di-sodium hydrogen phosphate (Qualikems Fine Chemicals, India), sodium acetate (Scharlau Chemie, Spain), and carrageenan K (SigmaAldrich, USA). All organic solvents used were of high-performance liquid chromatography (HPLC) grade.

\section{Preparation of Ibuprofen Gel}

Chitosan gel was prepared by dissolving $1 \mathrm{~g}$ of chitosan powder in $0.1 \mathrm{M}$ sodium acetate buffer ( $\mathrm{pH} 4.5)$, to give a final concentration of $5 \% \mathrm{w} / \mathrm{w}$, with continuous mixing using a magnetic stirrer until a homogenous gel was formed. The gel was set aside for few minutes until the bubbles disappeared. Ibuprofen $(0.05 \mathrm{~g})$ was mixed with propanol to a level of $5 \% \mathrm{w} / \mathrm{w}$ and incorporated into the gel. Six different formulations (F1 - F6) were prepared (in addition to the blank and reference product) by incorporating different permeation enhancers to the final gel, as shown in Table 1. The gels were kept in plastic well-closed containers and stored at room temperature until the time of analysis. 
Table 1: Composition of the ibuprofen gel formulations

\begin{tabular}{|c|c|c|c|c|c|c|c|c|}
\hline \multirow[b]{2}{*}{ 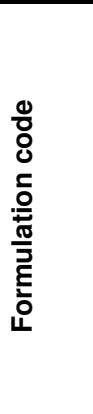 } & \multicolumn{8}{|c|}{ Formulation ingredients } \\
\hline & 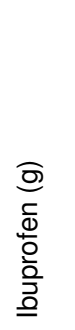 & 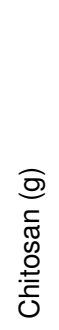 & $\begin{array}{l}\widehat{\jmath} \\
\frac{3}{0} \\
0 \\
\overline{0} \\
\frac{0}{\pi} \\
\overline{0} \\
\overline{0}\end{array}$ & $\begin{array}{c}\widehat{3} \\
3 \\
0 \\
0 \\
0 \\
0\end{array}$ & 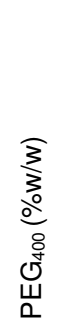 & $\begin{array}{l}\widehat{3} \\
30 \\
0 \\
\frac{0}{0} \\
0 \\
\frac{0}{0} \\
\overline{0}\end{array}$ & $\begin{array}{l}\widehat{3} \\
3 \\
0 \\
0 \\
\overline{0} \\
\frac{0}{0} \\
\frac{0}{0}\end{array}$ & $\begin{array}{l}\hat{3} \\
3 \\
0 \\
0 \\
\overline{0} \\
\frac{0}{1} \\
\frac{1}{1} \\
\sum^{0}\end{array}$ \\
\hline $\mathrm{F} 1$ & 0.05 & 1.0 & - & - & - & - & - & - \\
\hline $\mathrm{F} 2$ & 0.05 & 1.0 & 5.0 & - & - & - & $\begin{array}{c}5 . \\
0\end{array}$ & - \\
\hline F3 & 0.05 & 1.0 & 5.0 & $\begin{array}{l}2 \\
0\end{array}$ & - & - & - & - \\
\hline $\mathrm{F} 4$ & 0.05 & 1.0 & 5.0 & - & - & - & - & $\begin{array}{c}5 . \\
0\end{array}$ \\
\hline $\begin{array}{l}\text { F5 } \\
\text { F6 } \\
\text { Blank } \\
\text { lbutop }\end{array}$ & $\begin{array}{c}0.05 \\
0.05 \\
- \\
-\end{array}$ & $\begin{array}{c}1.0 \\
1.0 \\
1.0 \\
-\end{array}$ & $\begin{array}{c}5.0 \\
5.0 \\
- \\
-\end{array}$ & $\begin{array}{l}- \\
- \\
- \\
-\end{array}$ & $\begin{array}{c}3.0 \\
- \\
- \\
-\end{array}$ & $\begin{array}{c}- \\
1.0 \\
- \\
-\end{array}$ & $\begin{array}{l}- \\
- \\
-\end{array}$ & $\begin{array}{l}- \\
- \\
- \\
-\end{array}$ \\
\hline
\end{tabular}

\section{Determination of gel viscosity}

The viscosity of the formulations and reference was performed using a Brookfield digital viscometer (Model DV-II, USA) equipped with spindle S27. The apparent viscosity was measured at 17 seconds $^{-1}$ shear rate $(50 \mathrm{rpm})$ and room temperature, after a 3-min rest time [11].

\section{Determination of gel $\mathrm{pH}$}

One gram each of the gel formulations and the reference was accurately weighed and dispersed in $10 \mathrm{ml}$ of purified water. The $\mathrm{pH}$ of the dispersions was measured with a $\mathrm{pH}$ meter (Hanna Instruments, HI8417, Portugal) [12] .

\section{Evaluation of in vitro ibuprofen release}

A synthetic hydrophilic membrane was mounted on a Franz diffusion cell (PermeGear, Riegelsville, PA, USA). The receptor compartment contained $6.5 \mathrm{~mL}$ of phosphate buffer ( $\mathrm{pH} 7.4)$. One gram of the test formulation or reference was applied to the membrane over an area of $1.131 \mathrm{~cm}^{2}$ area across the donor compartment. The donor cell was exposed to ambient temperature and covered with parafilm to prevent evaporation. The temperature of the receptor compartment was maintained at 32 C while the buffer solution was stirred continuously with a Teflon-coated magnetic bar. Samples $(0.5 \mathrm{ml})$ were withdrawn from the release medium at $0,0.5,1,1.5,2,3,4$, 6 , and $8 \mathrm{~h}$ and replaced with an equal volume of fresh buffer solution to maintain sink conditions. The samples were analyzed by high performance liquid chromatography (HPLC) using the operating parameters described below (see 'HPLC analysis').

Cumulative amount of the drug permeated versus time (Zero order) and also versus square root of time (Higuchi model, Eq. 1 ) were obtained [13].

$$
Q=2 C_{\circ}\left(\frac{D T}{\pi}\right)^{\frac{1}{2}}
$$

where $\mathrm{C}_{\circ}=$ initial drug concentration in the donor compartment; $\mathrm{Q}=$ cumulative amount of the drug released at time, $t$; and $D=$ diffusion coefficient.

\section{HPLC analysis}

Analysis of ibuprofen in samples was performed using a Waters HPLC system equipped with a 1515 pump, 2487 detector, $717 \mathrm{P}$ auto-samplers and C18 column (5.0 $\mu \mathrm{m}, 3.9 \mathrm{~mm} \times 150 \mathrm{~mm}$ ). The mobile phase used was a mixture of $0.01 \mathrm{M} \mathrm{H}_{3} \mathrm{PO}_{4}$ and acetonitrile (55: 45). The flow rate was adjusted to $1 \mathrm{ml} / \mathrm{min}$ and the injection volume was $20 \mu \mathrm{L}$. The ultraviolet (UV) detector was set at a wavelength of $264 \mathrm{~nm}$ [14] and the retention time was $7.129 \mathrm{~min}$.

\section{Conditioning of the animals}

The experiments were conducted using female albino mice weighing 32 - $34 \mathrm{~g}$, taken from the Central Animal House, Dubai Pharmacy College, UAE. The animals were kept in plastic cages with soft bedding (6 per 
cage) under standard conditions of light and dark cycle and had free access to food and tap water. They were allowed to acclimatize for one week before the experiments. Ethical approval was given for the animal skin studies by the Research Ethics Committee of Dubai Pharmacy College and animal experiments were conducted in accordance with the "Guide for the Care and Use of Laboratory Animals" (NIH Publication no. 8523) [15].

\section{Skin irritation test}

This test was performed in accordance with ASTM method F719 - 81 [16]. A group of three male albino rabbits weighing approximately $1 \mathrm{~kg}$ were used in the test. The ventral side of the animal was carefully shaved, and six circular areas of $2.5 \mathrm{~cm}$ in diameter were drawn on the animal's abdomen. The circumscribed areas were painted with $20 \%$ aqueous solution of formaldehyde. for lipid drying and the solution was allowed to evaporate. Approximately 0.5 $\mathrm{g}$ of the formulations $\mathrm{F} 1, \mathrm{~F} 2, \mathrm{~F} 3, \mathrm{~F} 4$ and ibuprofen powder were placed on five of the circular areas and a substance of known irritancy (histamine) was injected intradermally into the sixth circular area for the purpose of calibration. The back of the animal's ear was shaved carefully; a piece of cotton was immersed in a small amount of xylene and applied to the shaved portion in order to dilate the superficial ear vein. One millilitre of $0.5 \%$ trypan blue was slowly injected into the selected vein and the degree of the resulting irritancy was estimated by the level of accumulation of trypan blue at the treated site. The degree of blueness was visually ranked to provide a relative order of irritancy of the substance used. The observations were made after 1, 6 and $24 \mathrm{~h}$.

\section{Evaluation of carrageenan-induced paw oedema}

Animals were divided into eight groups with eight animals in each; the first three groups served as controls: (a) un-inflamed, injected with saline; (b) inflamed, injected with carrageenan; (c) inflamed, treated with the reference product. The other five groups were inflamed and treated with the test ibuprofen formulations F1, F2, F3 and F4, and blank. Carrageenan solution ( $1 \% \mathrm{w} / \mathrm{v}$, in normal saline) was used to induce inflammation. The animals were placed singly in observation chambers for $10 \mathrm{~min}$ to minimize any stress-related behavioral changes. The mice then received sub-plantar administration of $50 \mu \mathrm{l}$ of the carrageenan solution in the left hind paw and were returned immediately to the observation chamber. The thickness $(\mathrm{mm})$ of the paw was measured at $0,1,2,3$ and $4 \mathrm{~h}$ after carrageenan administration, using an electronic digital caliper (Traceable Calipers, Friendswood, TX, USA) [17].

The gel formulations or the reference were applied to the plantar surface of the left hind paw by gently rubbing $0.5 \mathrm{~g}$ of the formulation 50 times with the index finger. Thirty minutes later, pleurisy was induced by injecting $50 \mu \mathrm{l}$ of $1 \% \mathrm{w} / \mathrm{v}$ carrageenan solution subcutaneously into the sub-plantar surface of the left paw of the mice. Control un-inflamed animals received $50 \mu \mathrm{l}$ normal saline subcutaneously into the sub-plantar surface of the left paw [18].

\section{Determination of analgesic activity}

Mice were divided into eight groups with eight animals per each and the test was carried out by the hot plate method [18]. The animals were habituated twice to the hot-plate in advance. The first three groups served as controls: (a) un-inflamed, injected with saline; (b) inflamed, injected with carrageenan; (c) inflamed, treated with the reference product. The other five groups were inflamed and treated with the test ibuprofen formulations F1, F2, F3 and F4, and blank. Thirty minutes prior to treatment, $1 \%$ carrageenan suspension $(0.01 \mathrm{ml})$ was injected into the subplanter area of the right hind paw In the test, the mice were placed on a hot plate maintained at $55 \pm 0.5{ }^{\circ} \mathrm{C}$. The time elapsed 
until the occurrence of either a hind paw licking or a jump off the surface was recorded as the hot-plate latency. The animals were tested at 0 (baseline), 0.5, 1.0, 1.5, 2.0, 2.5 and $3.0 \mathrm{~h}$. The cut-off time was $22.5 \mathrm{~s}$ to prevent tissue damage and mice with baseline latencies of $<5$ were eliminated from the study.

\section{Data analysis}

The results were expressed as mean \pm standard deviation. Analysis of variance (ANOVA) was performed for multiple comparisons followed by Bonferroni's multiple comparison. A level of significance of $p<$ 0.05 was set to determine any significant difference between the reference and developed formulations.

\section{RESULTS}

\section{Viscosity of gel formulations}

The results showed that there was no significant difference $(p<0.05)$ between the viscosities of gel formulations F2 - F6 and that of the reference. The addition of propanol produced a significant increment in the apparent viscosity of the prepared gels, compared with $\mathrm{F} 1 \quad(p<0.05)$, as shown in Table 2.

Table 2: Viscosity, release rate constant and correlation coefficient $\left(r^{2}\right)$ of the formulation

\begin{tabular}{llll}
\hline $\begin{array}{l}\text { Formulation } \\
\text { code }\end{array}$ & $\begin{array}{l}\text { Viscosity } \pm \\
\text { SD (cp) }\end{array}$ & $\begin{array}{l}\text { Release } \\
\text { rate } \\
\text { constant } \\
\left(\boldsymbol{\mu g} / \mathbf{h r}^{-1 / 2}\right)\end{array}$ & $\mathbf{r}^{2}$ \\
\hline F1 & $67700 \pm 100$ & $58.65 \pm 1.88$ & 0.99 \\
F2 & $72200 \pm 122$ & $83.50 \pm 2.38$ & 0.99 \\
F3 & $72320 \pm 120$ & $61.76 \pm 1.98$ & 0.99 \\
F4 & $72355 \pm 111$ & $86.00 \pm 3.45$ & 0.99 \\
F5 & $71900 \pm 100$ & $45.94 \pm 3.42$ & 0.96 \\
F6 & $72860 \pm 125$ & $44.22 \pm 3.69$ & 0.95 \\
Blank & - & - & - \\
lbutop ${ }^{\circledR}$ & $72500 \pm 123$ & $91.72 \pm 4.00$ & 0.99 \\
\hline
\end{tabular}

\section{In vitro drug release}

Ibuprofen release profiles over time from the various formulations and the reference are shown in Fig 1. The regression data for two kinetic models - zero order and Higuchi indicate that the data fitted better to the Higuchi model as indicated by higher $r^{2}$ values. The plot of the cumulative ibuprofen permeation through the synthetic membrane versus square root of time (not shown) was linear, thus indicating matrix diffusioncontrolled release kinetics. The drug release rate constants of the test formulations are presented in Table 2.
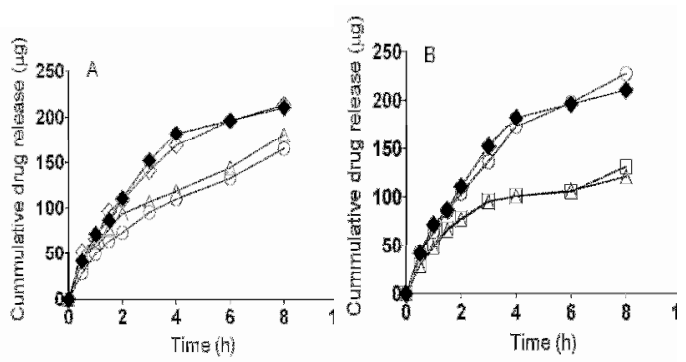

Figure 1: In vitro drug release profiles of $(\mathrm{A}) \mathrm{gel}$ formulations F1 (०), F2 (口), F3 $(\Delta)$ and Ibutop ( $\bullet$ ); and (B) gel formulations F4 (०), F5 (口), F6 $(\Delta)$ and Ibutop ( $\bullet$.

The release rate constants of ibuprofen from the various formulations follow the rank order: $\mathrm{F} 4>\mathrm{F} 2>\mathrm{F} 3>\mathrm{F} 1>\mathrm{F} 5>\mathrm{F} 6$, thus indicating that incorporation of menthol, glycerol and propylene glycol significantly $(p<0.05)$ increased drug release rate constants. In contrast,, drug release rate constant was not affected by the incorporation of either oleic acid nor PEG $400(p>0.05)$. There was, however, no significant difference $(p>0.05)$ in drug release between F1, F2, F3 and F4.

\section{Gel irritancy}

Ibuprofen-chitosan gel formulations were yellow in colour, homogeneous in texture and fell within a $\mathrm{pH}$ range of 4.5 to 5.0 which is compatible with normal skin $\mathrm{pH}$ in healthy people [19]. The results showed that after injection of trypan blue solution, the histamine 
site became gradually blue with the maximum intensity of blueness occurring after $6 \mathrm{~h}$. The intensity of blueness at the histamine site served as reference to other sites. The intensity of blueness increased only at the histamine site only, and hence the test gels were considered non-irritant.

\section{Anti-inflammatory activity}

The results (mice paw size) of the antiinflammatory test are shown in Fig 2. There was no significant difference between the anti-inflammatory effects of the test formulations and the reference and also the inflamed group ( $p>0.05)$ over the duration of the test.

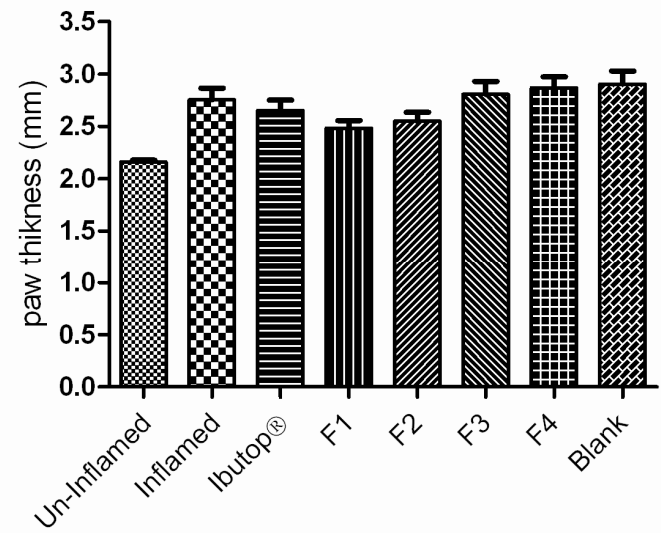

Figure 2: Anti-inflammatory effect of the test formulations, reference and control in carrageenan-induced paw oedema in mice (Error bar represents standard deviation)

\section{Analgesic effect}

The results of the assessment of analgesic activity are shown in Fig 3. Formulation F4 (containing menthol as permeation enhancer) showed a higher analgesic effect than the reference $(p<0.01)$. F3 formulation, with propylene glycol as permeation enhancer, also exhibited a significantly greater analgesic effect than the reference product ( $p$ $<0.05)$. F1 and F2 did not show significant analgesic effect $(p>0.05)$. Chitosan, which was the gel polymer used (represented by blank), did not show significant analgesic or anti-inflammatory activities $(p>0.05)$.

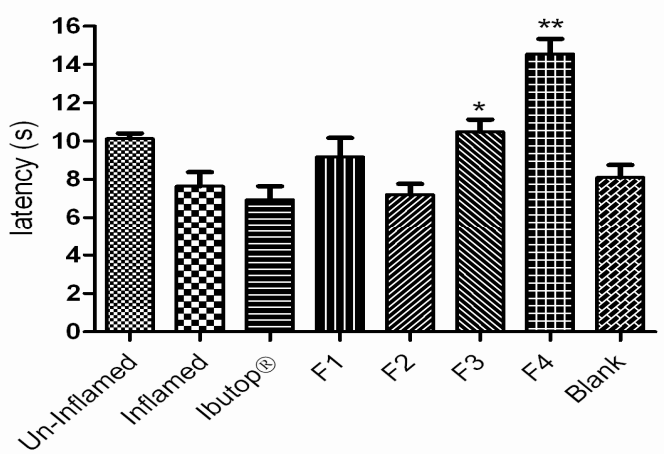

Figure 3: The analgesic effect on mice (measured by latency) of the different formulations using the hot plate method. ( ${ }^{* *} P<0.01$, ${ }^{*} P<0.05$; error bar represents standard deviation)

The analgesic effect of the preparations in relation to time is illustrated in Fig 4. Saline injection had no effect on paw withdrawal latencies (PWLs). A high significant $(p<0.01)$ reduction in PWLs compared to that of the reference was observed immediately after the application of formulation F4 which persisted for $2.5 \mathrm{~h}$. Similarly, a significant decrease in PWLs occurred followed application of formulation F3 but this was after a lag of $1 \mathrm{~h}$ and it continued for $2.5 \mathrm{~h}(p<0.01)$.

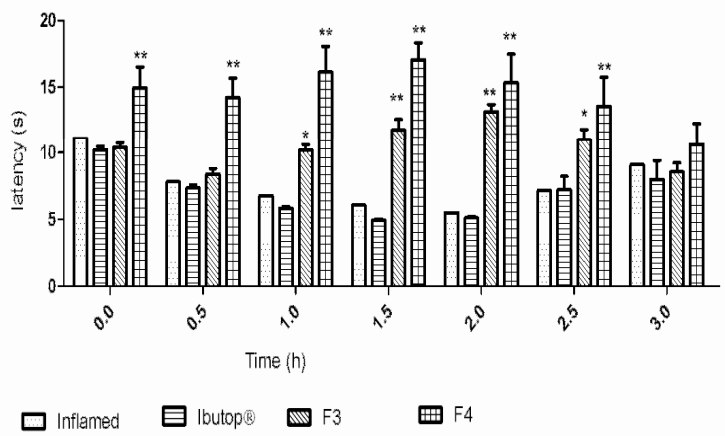

Figure 4: Effect of selected formulations on time course of carrageenan-induced hyperalgesia in mice $\left({ }^{*} P<0.01,{ }^{*} P<0.05\right.$; error bar represents standard deviation) 


\section{DISCUSSION}

The aim of the study is to develop ibuprofen transdermal gel formulations using various permeation enhancers and chitosan as gelling agent.

\section{Gel viscosity}

The apparent viscosity of the test formulations was comparable to that of the reference standard, lbutop ${ }^{\circledR}$. However, the inclusion of propanol increased the apparent viscosity of the test gels. This effect of propanol may be due to the association of the hydrophobic parts of the polymer (chitosan) with the alcohol, thus minimizing hydrophobic contact with water. The polymer-alcohol complex probably became more hydrophilic, thus making water a better solvent for the polymer. This complex might have expanded more in water and hence the increase in solution viscosity [20].

\section{Ibuprofen release}

In vitro drug release data from the test formulations fitted better to the Higuchi model than to the zero order model, an indication that the former more suitably described ibuprofen release kinetics from the test formulations and that drug release was matrix-diffusion controlled. The use of menthol, glycerol and propylene glycol (PG) as permeation enhancers significantly increased drug release rate constants compared to $\mathrm{F} 1$ which had no permeation enhancer. It has previously been shown that ibuprofen solubility is enhanced by the addition of menthol, due probably to the formation of a eutectic mixture of ibuprofen and menthol in aqueous solution [21]. On the other hand, glycerol and PG enhanced drug solubility by co-solvency effect [22].

It is generally agreed that in vitro drug release data for transdermal formulations cannot be used to accurately predict permeation across the skin, due to the barrier properties of the stratum corneum which can be altered by the composition of vehicle or the presence of various permeation enhancers in the formulation [23].

\section{Anti-inflammatory and analgesic properties of the gel formulations}

The results showed that no significant antiinflammatory effect was shown either by the test formulations or the standard reference, lbutop $^{\circledR}$. It may be that higher ibuprofen doses are required to produce in vivo antiinflammatory activity [24].

On the other hand, F4, which contained menthol as permeation enhancer, showed a higher analgesic effect than that of the reference standard. Some studies have reported that menthol is capable of acting as a skin penetration enhancer for a number of NSAIDs via different mechanisms such as induction of lipid packing disruption and formation of eutectic mixtures [25]. In addition to its well-known cooling effect via stimulation of skin's cold receptors [26], it also stimulates an axon reflex with subsequent release of vasodilator peptides [27] which may explain the high analgesic effect of the test formulation containing menthol.

Formulation F3 (with propylene glycol as permeation enhancer) also exhibited a higher analgesic activity than the reference. This could be explained by the fact that propylene glycol (PG) can penetrate the stratum corneum in a manner proportional to its level in the applied formulation and appears to alter ibuprofen solubility in the barrier [28]. Moreover, PG possibly infiltrates the outermost layer of the intercellular spaces of the stratum corneum and because most of the water accumulates within the corneocytes, it seems likely that $P G$ withdraws water from the cells due to its hygroscopic nature, causing skin hydration and thus enhances drug penetration [24]. 


\section{CONCLUSION}

New transdermal ibuprofen-chitosan gel formulations were developed successfully using different permeation enhancers and also propanol as a viscosity enhancing agent. Formulations containing $5 \%$ menthol and $20 \%$ propylene glycol demonstrated superior analgesic effect to the reference product, lbutop ${ }^{\circledR}$ in mice. However, the formulations did not show anti-inflammatory properties.

\section{ACKNOWLEDGEMENT}

The authors wish to express their gratitude to these undergraduate students: Noor Mudhaffer, Shamsa Ahmed, Walaa, Saleem, Amal Barakat, Fatema Al Tenaiji, Zakieh Mohammah Aqsa Abdul, Amina Ali, and Neda Ali who assisted in the course of this work.

\section{REFERENCES}

1. Daniels R, Knie U. Galenics of dermal productsvehicles,properties and drug release. JDDG 2007; 5: 367-381.

2. Babar A, Solanki UD, Cutie AJ, Plakogiannis F. Piroxicam release from dermatological bases: in vitro studies using cellulose membrane and hairless mouse skin. Drug Dev Ind Pharm 1990; 16: 523-540.

3. Busson MJ. Update on ibuprofen: review article. $J$ Int Med Res 1986;14:53-62.

4. Brown MB, Hanpanitcharoen M, Martin GP. An in vitro investigation into the effect of glycosaminoglycans on the skin partitioning and deposition of NSAIDs. Int J Pharm 2001; 225: 113-121.

5. Chen $H$, Chang $X$, Du D, Li J, Xu H, Yang $H$. Microemulsion-based hydrogel formulation of ibuprofen for topical delivery. Int $J$ Pharm 2006; 315: 52-58.

6. Brain KR, Green DM, Dykes PJ, Marks R, Bola TS. The Role of Menthol in Skin Penetration from Topical Formulations of Ibuprofen 5\% in vivo. Skin Pharmacology and Physiology 2005; 19: 17-21.

7. Park E, Chang S, Hahn M, Chi S. Enhancing effect of polyoxyethylene alkyl ethers on the skin permeation of ibuprofen. Int J Pharm 2000; 209: 109-119.

8. Kas HS. Chitosan: properties, preparations and application to micro-particulate systems. J Microencapul 1997; 14: 689-711.
9. Hiroshi $H$, Keta W, Ratana RV, Seiichi T. Preservation of chitosan aqueous gel under neutral conditions. J Metals Materials and Minerals 2005; 15: 19-21.

10. Awasthi S, Irshad M, Das MK, Ganti SS, Moshahid A.Rizvi. Anti-Inflammatory Activity of Calotropis gigantea and Tridax procumbens on Carrageenin-Induced Paw Edema in Rats. Ethnobotanical Leaflets 2009; 13: 568-577.

11. Dai Y, But PP, Matsuda H, Kubo M. Antipruritic and Anti-inflammatory effects of aqueous extract from Si-Wu-Tang. Biol Pharm Bull 2002; 25 : 1175-1178

12. Mario J, Mira B.L., Biserka C.C. Influence of Cyclodextrin Complexation on Piroxicam Gel Formulaion. Acta Pharma 2005; 55: 223-236.

13. Ozsoy Y, Gunfor S, Cevher E. Vehicle effects on in vitro release of Tiaprofenic acid from different topical formulations. IL Farmoaco, 2004;59:563-566.

14. Kofuji K, Akamine H, Qian CJ, Watanabe K, Togan $Y$, Nishimura $M$, Sugiyama I, Murata $Y$, Kawashima S. Therapeutic Efficacy of Sustained Drug Release from Chitosan Gel on Local Inflammation. Int J Pharm 2004; 272: 6578.

15. ASTM F719 - 81. Standard practice for testing biomaterials in rabbits for primary skin irritation. West Conshohocken, Philadelphia; 2007: 178-179.

16. US National Institutes of Health. Guide for the care and use of laboratory animals (NIH Publication No 85-23), 1996.

17. Winter CA, Risley EA, Nuss GW. Carrageenaninduced edema in hind paws of the rat as an assay for anti-inflammatory drugs. Proc Soc Exp Bio Med 1962; 111: 544-547.

18. Adzu B, Amos S, Kapu SD, Gamaniel KS. Antiinflammatory and antinociceptive effects of Sphaeranthus senegalensis. Journal of Ethnopharmacology 2003; 84: 169-173.

19. Hadgraft J. Skin, the final frontier. Int J Pharm 2001; 224: 1-18.

20. Wu N, Parris J. Interaction of water-soluble acrylic polymers with alcohols in aqueous solutions. Colloids and Surfaces A: Physicochemical and Engineering Aspects 2000; 179: 167-187.

21. Miguel R. Eutetic mixture of local anesthetics: not just for kids. Reg Anesth 1994; 19: 75-76.

22. Kashappa GH., Park HJ. Solubility studies on valdecoxib in the presence of carriers, cosolvents, and surfactants. Drug Dev Res 2004; 62: 41-48.

23. Sinha VR, Maninder PK. Permeation enhancers for transdermal drug delivery. Drug Dev Ind Pharm 2000; 26: 1131-1140.

24. Bradley $J D$, Brandt $K D$, Katz $B P$, Kalasinski $L A$, Ryan SI. Comparison of an anti inflammatory dose of ibuprofen, an analgesic dose of ibuprofen, and acetaminophen in the treatment of patients with osteoarthritis of the knee. New Engl J Med 1991; 325: 87-91. 


\section{Rasool et al}

25. Stott PW, Williams AC, Barry BW. Transdermal delivery from eutectic systems: enhanced permeation of a model drug, ibuprofen. $J$ Controlled Rel 1998; 50: 297-308.

26. David AA, Henry WN, Stuart B. TRPM8 activation by menthol, icilin, and cold is differentially modulated by intracellular $\mathrm{pH}$. J Neurosci 2004; 24: 5364-5369.
27. Eccles R. Menthol and related cooling compounds. J Pharm Pharmacol 1994; 46: 618-630.

28. Herkenne $C$. Effect of propylene glycol on ibuprofen absorption into human skin in vivo. J Pharm Sci 2008; 97: 185-197. 